\title{
Lymphopenia and Immune Deficiency: Eyes See Only What the Mind Knows!
}

\author{
Sagar Bhattad \\ Pediatric Infectious Disease (2019): 10.5005/jp-journals-10081-1223
}

\section{INTRODUCTION}

Complete blood count (CBC) is a commonly requested investigation in clinical practice. High white cell count (WBC) is often presumed to be marker of an underlying infection. We often fail to make a note of the differential counts, which, in fact, provide more valuable information than total WBCs. Clinicians are tuned to make a note of high neutrophil counts, and it is common to consider "sepsis" as a possibility in a febrile child with high neutrophil counts. But do you regularly make a note of the absolute lymphocyte counts (ALCs)? Have you noted children with persistently low lymphocyte counts and wondered what is going on? Lymphopenia can often be a marker of an underlying sinister disease!

\section{LYMPHOCYTES}

Lymphocytes are white blood cells that play a crucial role in defense against microorganisms. They also take part in eliminating cancer cells (cancer surveillance) and rejecting organs after transplants. T cells, B cells, and natural killer (NK) cells are the three types of lymphocytes. Cell-mediated immunity is proffered by T cells, while B cells produce immunoglobulins and provide the humoral immunity. Natural killer cells form a part of the innate immune defense against viruses and tumors.

Grossly, T cells form $70 \%$ of the peripheral blood lymphocytes, while B and NK cells form 20 and 10\%, respectively. Absence or severe reduction in $T$ cell counts would thus result in absolute lymphopenia (while low B cell or NK cell counts would not do so). Thus, in any patient presenting with severe and persistent lymphopenia, T cell lymphopenia is likely.

It is also important to note that B cells need help from CD4+ $T$ cells for their final maturation and production of high-affinity antibodies. Any T cell defect would thus result in B cell dysfunction, and this is what we call the "combined immune deficiency" (CID).

\section{Healthy Infants Have High Lymphocyte COUNTS}

Lymphocytic predominance is physiological in infancy. Unlike adults, where neutrophils contribute to $70 \%$ of peripheral blood leukocytes, lymphocytes contribute to around 50 to $55 \%$ of leukocytes in infants. Lymphocyte counts of less than $3,000 / \mathrm{mm}^{3}$ in infancy warrants immediate attention!

\section{LYMPHOPENIA}

Lymphopenia can be caused by viral infections, exposure to glucocorticoids, autoimmune diseases like systemic lupus erythematosus, etc. However, it is transient in these conditions, and
Department of Pediatric Immunology and Rheumatology, Aster CMI Hospital, Bengaluru, Karnataka, India; Foundation for Primary, Immunodeficiency Diseases (USA) Center, Aster CMI Hospital, Bengaluru, Karnataka, India

Corresponding Author: Sagar Bhattad, Department of Pediatric Immunology and Rheumatology, Aster CMI Hospital, Bengaluru, Karnataka, India; Foundation for Primary, Immunodeficiency Diseases (USA) Center, Aster CMI Hospital, Bengaluru, Karnataka, India, Phone: +91 9779433934, e-mail: drsagarbhattad@gmail.com

How to cite this article: Bhattad S. Lymphopenia and Immune Deficiency: Eyes See Only What the Mind Knows! Pediatr Inf Dis 2019;1(4):169-170.

Source of support: Nil

Conflict of interest: None

\begin{tabular}{ll}
\hline Age & ALC \\
\hline $0-2$ years & $<3,000 / \mathrm{mm}^{3}$ \\
2-6 years & $<1,500 / \mathrm{mm}^{3}$ \\
$>6$ years & $<1,000 / \mathrm{mm}^{3}$ \\
\hline
\end{tabular}

lymphocyte counts tend to normalize once the trigger is removed or treated. Presence of lymphopenia on two or more occasions in the first 2 years of life must make one think of severe combined immune deficiency (SCID).

\section{LYMPHOPENIA AND SCID}

Severe combined immune deficiency is a severe form of immune deficiency characterized by the absence of $T$ cells since birth. Children present with opportunistic infections (Pneumocystis jiroveci pneumonia, candidiasis, and disseminated cytomegalovirus infection), chronic diarrhea, and failure to thrive in early infancy. This disease is universally fatal, and children die within the first 2 years of life unless definitive treatment is provided. Severe combined immune deficiency is a medical emergency and warrants immediate referral to a center with expertise in the management of these children. Bone marrow transplant is curative and majority of the children can be cured if diagnosed and treated early. What is essential to understand is that the clue to such a serious disease lies in the CBC. These children have persistent lymphopenia and can be easily diagnosed, provided one is aware of these diseases. 


\section{Definition of SCID}

Male or female patient less than 2 years of age with less than $20 \%$ CD3+ T cells, an ALC of less than $3,000 / \mathrm{mm}^{3}$, and mutation in a gene are known to cause SCID (as per the European Society for Immunodeficiencies).

\section{Case Description}

A 4-month-old girl born to a third-degree consanguineous couple presented with persistent oral thrush and failure to thrive. She had an episode of pneumonia and was hospitalized. Parents reported death in the first two children at 3 and 5 months, respectively, likely due to sepsis.

On examination, she was malnourished and tachypneic.

Evaluation: Chest X-ray-no thymic shadow.

\section{CBC}

\begin{tabular}{lllll}
\hline $\mathrm{HB}(\mathrm{g} / \mathrm{dL})$ & 9 & 8.5 & 8.3 & 8.2 \\
WBC (per mm $\left.\mathrm{mm}^{3}\right)$ & 9,100 & 11,000 & 10,800 & 8,900 \\
DC & $\mathrm{N}_{86} \mathrm{~L}_{5} \mathrm{M}_{5} \mathrm{E}_{4}$ & $\mathrm{~N}_{80} \mathrm{~L}_{8} \mathrm{M}_{7} \mathrm{E}_{5}$ & $\mathrm{~N}_{88} \mathrm{~L}_{5} \mathrm{M}_{5} \mathrm{E}_{2}$ & $\mathrm{~N}_{78} \mathrm{~L}_{9} \mathrm{M}_{7} \mathrm{E}_{6}$ \\
ANC $\left(\right.$ per mm $\left.\mathrm{mm}^{3}\right)$ & 7,826 & 8,800 & 9,504 & 6,942 \\
ALC $\left(\right.$ per mm $\left.\mathrm{mm}^{3}\right)$ & 455 & 880 & 540 & 801 \\
PC (per $\left.\mathrm{mm}^{3}\right)$ & 130,000 & 145,000 & 123,000 & 134,000 \\
\hline
\end{tabular}

$\mathrm{HB}$, hemoglobin; DC, differential counts; ANC, absolute neutrophil counts; $\mathrm{PC}$, platelet counts

There was persistent lymphopenia!

Immunological tests were carried out-lgG $<130 \mathrm{mg} / \mathrm{dL}$, IgA $<30 \mathrm{mg} / \mathrm{dL}$, and $\mathrm{lgM}<20 \mathrm{mg} / \mathrm{dL}$ (serum immunoglobulins were markedly reduced).

Lymphocyte subsets-CD3: 2\% (50-70\%), CD19: 91\% (10-15\%), and CD56: 3\% (5-10\%).

Markedly reduced $\mathrm{T}$ cells in this setting confirm a diagnosis of SCID.

Genetic testing - mutation in JAK3 gene.

\section{Diagnostic Algorithm}

Clinical suspicion (failure to thrive, recurrent infections in early infancy, and history of death in siblings)
Lymphopenia and absent thymus

$\downarrow$

Low T cells and hypogammaglobulinemia

$\downarrow$

Severe combined immune deficiency

\section{LYMPHOPENIA AND CID}

The presence of lymphopenia in children beyond the age of 2 and adults may point toward a CID. These patients often present with opportunistic infections and autoimmunity [e.g., idiopathic CD4 lymphocytopenia, activated PI3 kinase delta syndrome (APDS)].

\section{Case Description}

A 30-year-old man presented with recurrent pneumonia from 10 years of age and had been diagnosed to have bronchiectasis. $\mathrm{He}$ also had repeated episodes of herpes labialis. On examination, he had grade III clubbing, generalized lymphadenopathy, and splenomegaly.

Evaluation: All the previous records showed persistent lymphopenia $\left(A L C<1,000 / \mathrm{mm}^{3}\right.$ ). Immunoglobulin profile was normal. While T, B, and NK cell\% were normal, absolute counts were low. Genetic study (whole exome sequencing) showed a mutation in PIK3CD gene confirming the diagnosis of APDS.

Activated PI3 kinase delta syndrome is a recently described primary immune deficiency characterized with recurrent respiratory infections, herpes virus infections, malignancies, and autoimmunity.

\section{Message}

- Always look at the lymphocyte counts while evaluating sick infants.

- Persistent lymphopenia is a strong pointer toward SCID in infants.

- Children with SCID die in the first 2 years of life, unless they undergo a bone marrow transplant.

- Lymphopenia in older children and adults can be manifestation of CID. 